\title{
CROSSCAPS AND KNOTS
}

\section{BRADD EVANS CLARK}

\section{Introduction}

Seifert demonstrated in 1934 that every knot can be spanned by an orientable surface. These Seifert surfaces lead to numerous knot invariants. The purpose of this paper is to demonstrate the existence of a parallel theory concerning connected nonorientable surfaces. These surfaces give rise to additional knot invariants.

If $\mathrm{X}$ is a point set we let $\operatorname{cl}(\mathrm{X})$ stand for the closure of $\mathrm{X}$, int(X) stand for the interior of $\mathrm{X}$, and $\partial \mathrm{X}$ stand for the boundary of $\mathrm{X}$. If $S$ is a surface, let $\chi(S)$ stand for the Euler characteristic of $S$. If $\mathrm{K}$ is a knot in Euclidean 3-dimensional space, $E^{3}$, let $g(k)$ stand for the genus of $k$ as defined by Seifert in [4].

This paper deals with piecewise linear topology. As such, all manifolds and maps will be considered to be piecewise linear.

\section{The Crosscap Number}

Let $k$ be a knot in $E^{3}$, and $E^{2} \subset E^{3}$ a plane with $\pi: E^{3} \rightarrow E^{2}$ the orthogonal projection. We say that $E^{2}$ is regular for $k$ provided every $\pi^{-1}(x), \quad x \in E^{2}$ intersects $k$ in at most two points; and if 
$\pi^{-1}(x) \cap k$ contains two points, neither is a vertex of $k$. Reidemeister in [3] described the "checkerboard surfaces" associated with a regular projection of $\mathbf{k}$.

Theorem 2.1. If $\mathrm{k}$ is a nontrivial knot and $\mathrm{E}^{2}$ is any plane which is regular for $k$, then at least one of the as sociated checkerboard surfaces is nonorientable.

Proof: Let us call the checkerboard surfaces as sociated with $k$ and the orthogonal projection $\pi: E^{3} \rightarrow E^{2}, S_{1}$ and $S_{2}$. At each crossing of $k$, both $S_{1}$ and $S_{2}$ contain a disk which is twisted with respect to $\pi$. We place an orientation on $k$. Then one of the twisted disks must disagree with the orientation given $k$. Suppose that $S_{1}$ is the surface which contains that twisted disk.
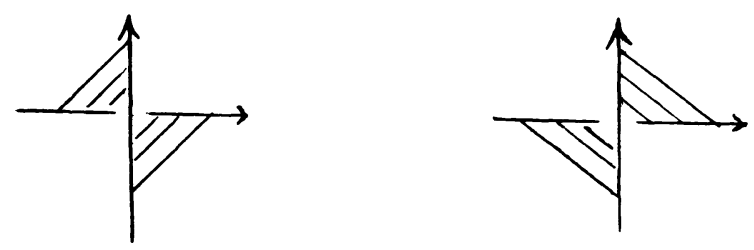

Figure 1

If $S_{1}$ is orientable, then we can use the orientation of the surface to reorient the knot. At the crossing under consideration, we 
would have to leave one arc of $k$ with the same orientation and another arc of $k$ would have to change its orientation. Since $k$ is a simple closed curve, this is a contradiction. Therefore $S_{1}$ is nonorientable. Consider a nonorientable surface $S$ which spans a knot $k$. If we were to sew a disk to $\partial S$, we would obtain a nonorientable closed 2-manifold. This manifold could be considered as the connected sum of $n$ projective planes. Let $C(S)=1-\chi(S)=n$.

Definition: The crosscap number of a knot $k, C(k)$, is the minimum value for $C(S)$ where $S$ stands for any nonorientable surface spanning $k$ in $E^{3}$. For completeness we shall define $C(k)=0$ if and only if $k$ is the unknot.

Proposition 2.2. $C(k)=1$ if and only if $k$ is a $(2, n)$ cable knot.

Proof: If $\mathrm{C}(\mathrm{k})=1$, then there is a Mbbius band $\mathrm{B}$ which spans $k$. The centerline of $B$ is a knot, which makes $k$ a $(2, n)$ cable about that knot. If $\mathrm{k}$ is a $(2, \mathrm{n})$ cable about a knot, the construction of a spanning Mbbius band is clear.

A study of embeddings of Mbbius bands in $\mathrm{S}^{3}$ was made by Kyle in [2].

Corollary 2.3. There exist knots of arbitrarily large genus with $\mathrm{C}(\mathrm{k})=1$. 
If $S$ is a nonorientable surface spanning a knot $k$, we can think of $S$ as a disk $D$ and a collection of handles $\left\{h_{i}\right\}$. Aso, $D \cap h_{i}$ will consist of two arcs, $a_{i 1}$ and $a_{i 2}$. We can find an embedding $f$ of $D \times I$ into $S^{3}$ such that $f\left(D \times\left\{\frac{l}{2}\right\}\right)=D$ and $f(D \times I) \cap h_{i}=a_{i 1} U a_{i 2}$ for all $i$. We can also find a collection of embeddings $f_{i}$ of $h_{i} \times I$ such that $f_{i}\left(h_{i} \times\left\{\frac{1}{2}\right\}\right)=h_{i}, f_{i}\left(h_{i} \times I\right) \cap f_{j}\left(h_{j} \times I\right)=\emptyset$ if $i \neq j$, and $f(D \times I) \cap f_{i}\left(h_{i} \times I\right)=f\left(\left(a_{i 1} U a_{i 2}\right) \times I\right)=f_{i}\left(\left(a_{i 1} U a_{i 2}\right) \times I\right)$.

Definition: We define $D(S)$, the double of $S$, to be $\bigcup_{i=1}^{n} f_{i}\left(h_{i} \times\{0,1\}\right) \cup f(D \times\{0,1\})$.

Theorem 2.4. $D(S)$ is a connected orientable surface which has a cable link of two components about $k$ for its boundary and double covers $\mathrm{S}$.

Proof: It is clear that $D(S)$ double covers $S$ since it does so locally. Also $\mathrm{D}(\mathrm{S})$ is connected since $\mathrm{S}$ contains a nonorientable handle, which causes $D(S)$ to contain two handles connecting $f(D \times\{0\})$ to $f(D \times\{1\})$. Let $M=\bigcup_{i=1}^{n} f_{i}\left(h_{i} \times I\right) U f(D \times I)$. Then $M$ is a 3-manifold with boundary embedded in $S^{3} . D(S) \subset \partial M \subset S^{3}$, which implies that $D(S)$ is orientable. Also the knot $k \subset \partial M$. Since $\partial M$ is orientable, and $\partial M-D(S)$ is a regular neighborhood of $k$ in $\partial M$, $\partial M-D(S)$ is an annulus. So $\partial D(S)$ is a link. 
Let $\partial D(S)$ be the two simple closed curves $l_{1}$ and $l_{2}$. Clearly $l_{1}$ and $l_{2}$ are isotopic to $k$ and in fact are $(1, n)$ cables about $k$. The value of $n$ will depend upon the surface $S$.

Definition: A k-triple $\left(C_{n}, A, \pi\right)$ is a cube-with-n-holes, possibly knotted, $C_{n}$, a nonseparating annulus $A \subset \partial C_{n}$, and a fixed point free involution $\pi$ on $\operatorname{cl}\left(\partial C_{n}-A\right)$, such that identifying each point $x \in \operatorname{cl}\left(\partial C_{n}-A\right)$ with $\pi(x)$ will yield a cube-with-k-knotted hold.

Theorem 2.5. $C(k)=n$ if and only if the minimal number of holes needed for a k-triple is $n$.

Proof: Let $S$ be a nonorientable surface spanning $k$. We construct $D(S)$ as described above and find a regular neighborhood of $k$ in $S^{3}, N(k)$, with $\partial D(S) \subset \partial N(k)$ and $N(k) \cap S$ a collar for $k$ in $\mathrm{S}$.

$D(S)$ separates the cube-with-k-knotted hole, $c 1\left(S^{3}-N(k)\right)$, into two pieces. One piece is $\bigcup_{i=1}^{n} f_{i}\left(h_{i} \times I\right) U f(D \times I)$ which is a cube-with-n-handles. Since $\bigcup_{i=1}^{\pi} f_{i}\left(h_{i} \times I\right) U f(D \times I) U N(k)$ is homeomorphic to $\underset{i=1}{U} f_{i}\left(h_{i} \times I\right) U f(D \times I)$, we must have that $C_{n}=\operatorname{cl}\left(S^{3}-\left(\bigcup_{i=1}^{n} f_{i}\left(h_{i} \times I\right) U f(D \times I) U N(k)\right)\right)$ is a cube-with-n holes. $\partial C_{n}=D(S) U_{A}$ where $A$ is an annulus contained in $\partial N(k)$.

Since by Theorem 2.4, $\mathrm{D}(\mathrm{S})$ is connected, A must be a nonseparating annulus. 
Finally we note that $D(S)$ is a double cover of $S$. Let $P$ be the natural projection from $D(S)$ to $S$. If $x \in S$ we can find exactly two points $x_{1}, x_{2} \in D(S)$ such that $P\left(x_{1}\right)=P\left(x_{2}\right)=x$. Define $\pi: D(S) \rightarrow D(S)$ by $\pi\left(x_{1}\right)=x_{2}$ and $\pi\left(x_{2}\right)=x_{1}$. Clearly $\pi$ is a fixed point free involution on $\operatorname{cl}\left(\partial C_{n}-A\right)=D(S)$, and identifying $x \in D(S)$ with $\pi(x)$ will yield a cube-with-k-knotted hole. Thus $\left(C_{n}, A, \pi\right)$ is a $\mathrm{k}$-triple, and the number of holes in $C_{n}$ depends on the number of handles in $\mathrm{S}$. Thus the minimal number of holes needed for a $\mathrm{k}$-triple is less than or equal to $\mathrm{C}(\mathrm{k})$.

Now let $\left(C_{n}, A, \pi\right)$ be a k-triple. We perform the identification which yields a cube-with-k-knotted hole. We think of $k$ as lying in the boundary of the cube-with-k-knotted hole, and note that the image of $\operatorname{cl}\left(\partial C_{n}-A\right)$ is a 2-manifold $S$ which spans $k . C_{n}$ can be reconstructed by removing an open regular neighborhood of $S$ from the cube-with-kknotted hole. If $S$ were orientable, then the annulus $A$ from the $k$-triple will separate $\partial C_{n}$. Thus $S$ must be nonorientable and the number of holes in $C_{n}$ is greater than or equal to $C(k)$.

The importance of $\mathrm{k}$-triples to covering space theory will be demonstrated in the next section.

Mark Kidwell has pointed out that any orientable surface spanning a knot can be changed to a nonorientable surface by performing a Reidemeister move on the projection. By adding a trivial loop we can 
add a nonorientable handle to the surface. This means that the crosscap number of a knot is bounded by the genus of the knot.

Proposition 2.6. $\mathrm{C}(\mathrm{k}) \leq 2 \mathrm{~g}(\mathrm{k})+1$.

Proof: Let $S$ be an orientable surface of minimal genus spanning $k$. Then $\chi(S)=1-2 g(k)$. By changing the surface to a nonorientable surface as described above we obtain $S^{\prime}$ with $\chi\left(S^{\prime}\right)=-2 g(k)$. So $C\left(S^{\prime}\right)=2 g(k)+1$.

Question: Do there exist knots for which $C(k)=2 g(k)+1$ ? Could the alternating pretzel knots have this property?

Let $k_{1}$ and $k_{2}$ be knots. We let $k_{1} \# k_{2}$ stand for the "connected sum" of $k_{1}$ and $k_{2}$. Suppose that $s_{1}$ is a surface of maximal Euler characteristic spanning $k_{1}$ and $S_{2}$ is a surface of maximal Euler characteristic spanning $\mathrm{k}_{2}$.

Lemma 2.7. The maximal Euler characteristic for a surface spanning $\mathrm{k}_{1} \# \mathrm{k}_{2}$ is $\chi\left(\mathrm{S}_{1}\right)+\chi\left(\mathrm{S}_{2}\right)-1$.

A proof of this lemma can be found in [1].

Theorem 2.8. $\mathrm{C}\left(\mathrm{k}_{1}\right)+\mathrm{C}\left(\mathrm{k}_{2}\right)-1 \leq \mathrm{C}\left(\mathrm{k}_{1} \# \mathrm{k}_{2}\right) \leq \mathrm{C}\left(\mathrm{k}_{1}\right)+\mathrm{C}\left(\mathrm{k}_{2}\right)$.

Proof: Applying Lemma 2.5 to a nonorientable surface spanning $k_{1}$ and a nonorientable surface spanning $k_{2}$ gives us that 
$C\left(k_{1} \# k_{2}\right) \leq C\left(k_{1}\right)+C\left(k_{2}\right)$. In fact, if the se surfaces are of maximal Euler characteristic for those spanning $k_{1}$ and $k_{2}$, we get equality. However, if one of these knots, say $\mathrm{k}_{1}$, has $\mathrm{C}\left(\mathrm{k}_{1}\right)=2 \mathrm{~g}\left(\mathrm{k}_{1}\right)+1$, we can replace the nonorientable surface spanning $k_{1}$ by an orientable surface of maximal Euler characteristic. By Lemma 2. 5 we'd then have $C\left(k_{1} \# k_{2}\right)=C\left(k_{1}\right)+C\left(k_{2}\right)-1$.

\section{Covering Space Theory}

Seifert used his orientable spanning surfaces to form various covering spaces. In a like manner we shall use our nonorientable surfaces to form various covering spaces. As we saw in Theorem 2.5, each nonorientable spanning surface can be associated with a k-triple. Let $\left(C_{n}, A, \pi\right)$ and $\left(C_{n}^{\prime}, A^{\prime}, \pi^{\prime}\right)$ be identical copies of a k-triple. Let id $: \operatorname{cl}\left(\partial C_{n}-A\right) \rightarrow \operatorname{cl}\left(\partial C_{n}^{\prime}-A^{\prime}\right)$ be the identity map.

Theorem 3. 1. Identifying $x \in \operatorname{cl}\left(\partial \mathrm{C}_{\mathrm{n}}-\mathrm{A}\right)$ with $\pi^{\prime}(\mathrm{id}(\mathrm{x})) \in \operatorname{cl}\left(\partial \mathrm{C}_{\mathrm{n}}^{\prime}-\mathrm{A}^{\prime}\right)$ forms a two-fold cover $C$ of a cube-with-k-knotted hole K.

Proof: Obviously int $C_{n} U$ int $C_{n}^{\prime}$ is a double cover of int(K-S), $A \cup A^{\prime}$ is a double cover of $\partial K$, and $\operatorname{cl}\left(\partial C_{n}-A\right)=\operatorname{cl}\left(\partial C_{n}^{\prime}-A^{\prime}\right)$ is a double cover of $\mathrm{S}$. All we need to show is that the se double covers agree. Let $x \in S$ and $N(x)$ a ball about $x$ which is split into a left half and a right half by S. Then $N(x)$ will lift to a left half ball and a right half ball in $C_{n}$ and a left half ball and a right half ball in $C_{n}^{\prime}$. 
But $\pi^{\prime}(\operatorname{id}(N(x) \cap S))$ sews the left half ball of $C_{n}$ to the right half ball of $C_{n}^{\prime}$ and the right half ball of $C_{n}$ to the left half ball of $C_{n}^{\prime}$. So indeed we have a double cover of $\mathrm{K}$.

Of course, any covering space of $C$ is also a covering space of K. Thus we really have an infinite number of covering spaces associated with the nonorientable surface $\mathrm{S}$.

The $\mathrm{C}_{\mathrm{n}}$ in Theorem 3.1 is a fundamental region in our covering space. A similar situation occurs in Seifert's covering spaces. If $S$ is an orientable surface spanning $k$, then $N(S)$, a regular neighborhood of $\mathrm{S}$ in $\mathrm{K}$ will be a cube-with-handles. Thus a fundamental region in the usual cyclic coverings is also a cube-with-holes.

In this light, the question in Section 2, a sking if there is a knot $k$ with $C(k)=2 g(k)+1$, translates to "does there exist a knot $k$ all of whose double covers with fundamental region having the fewest possible number of holes are associated with orientable surfaces?"

Theorem 3.2. For any knot $k$, and any integer $n$, there exists a nonorientable surface $S$ such that $\partial D(S)$ has linking number $n$.

Proof: Let $l_{1}^{\prime}$ and $l_{2}^{\prime}$ be the boundary curves for $D\left(S^{\prime}\right)$ where $S^{\prime}$ is any nonorientable surface spanning the knot $k$. Then since $l_{2}^{\prime}$ is isotopic to $\mathrm{k}$ in the complement of $l_{1}^{\prime}$ in $\mathrm{S}^{3}$, the linking number of $\ell_{1}^{\prime}$ with $\ell_{2}^{\prime}$ is the same as the linking number of $\ell_{1}^{\prime}$ with $k$. 
We can change $k$ in an a rbitrarily small neighborhood by adding a trivial loop. This will change the surface $S^{\prime}$ to a surface $S$ which has one more handle and agrees with $S^{\prime}$ except in an a rbitrarily small neighborhood.

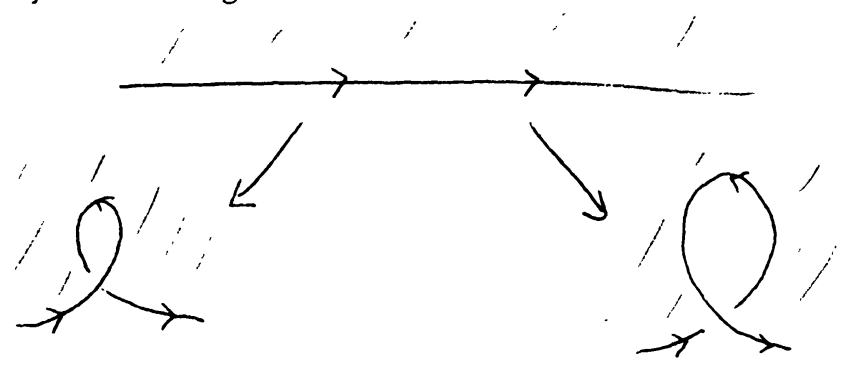

Figure 2

If $l_{1}$ and $l_{2}$ are the boundary components of $D(S)$, we have that $\ell_{1}$ and $\ell_{2}$ agree with $\ell_{1}^{\prime}$ and $\ell_{2}^{\prime}$ in all but an arbitrarily small neighborhood, and the linking number of $l_{1}$ with $l_{2}$ is either one more or one less than the linking number of $\ell_{1}^{\prime}$ with $\ell_{2}^{\prime}$.

This theorem shows that we can find a double cover of a cubewith-k-knotted hole which has a cube-with-n-holes for a fundamental region for any $n \geq C(k)$. 


\section{REFERENCES}

1. Fox, R. H. A Quick Trip Through Knot Theory, Topology of 3-Manifolds and Related Topics, M. K. Fort, Jr. editor.

2. Kyle, R. H. Embeddings of Mobius Bands in 3-Dimensional Space, Proc. Roy. Irish Acad. Sect A 57 (1955) 120-167.

3. Reidemeister, K. Knotentheorie, Chelsea Publishing Company, New York, 1948.

4. Seifert, H. Uber das Geschelect von Knoten, Math. Ann. 110 (1934) 571592.

Department of Mathematics

University of Southwestern Louisiana

Lafayette, Louisiana 70504 U.S.A.

(Received July 18, 1977 and in revised form April 3, 1978)

ABSTRACT. Seifert (4) demonstrated that every knot can be spanned by an orientable surface. These Seifert surfaces lead to numerous knot invariants. The purpose of this paper is to demonstrate the existence of a parallel theory concerning connected nonorientable surfaces. These surfaces give rise to additional knot invariants.

KEY WORDS AND PHRASES. Nonorientable surfaces, knot invariants, crosscaps and knots. 


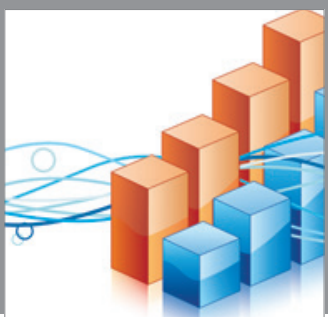

Advances in

Operations Research

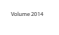

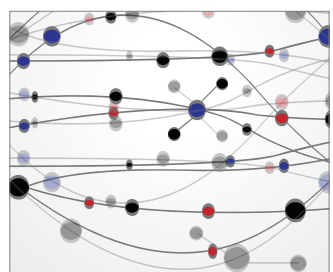

\section{The Scientific} World Journal
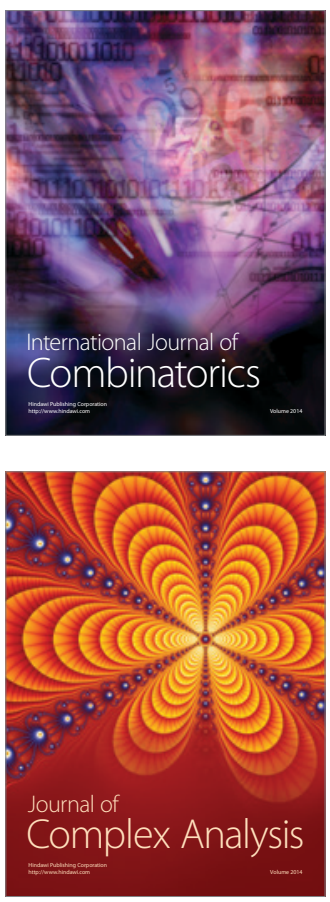

International Journal of

Mathematics and

Mathematical

Sciences
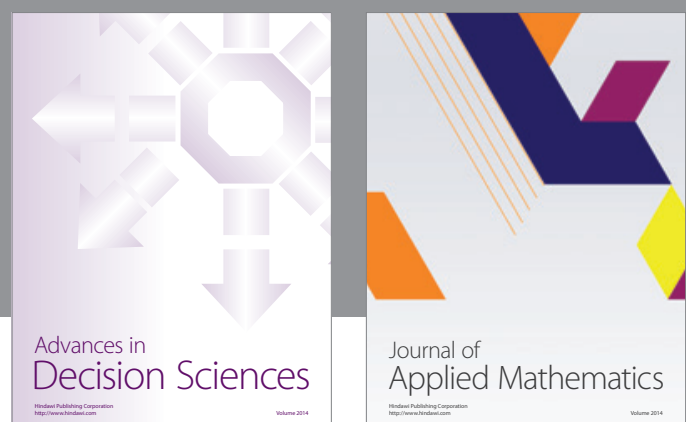

Journal of

Applied Mathematics
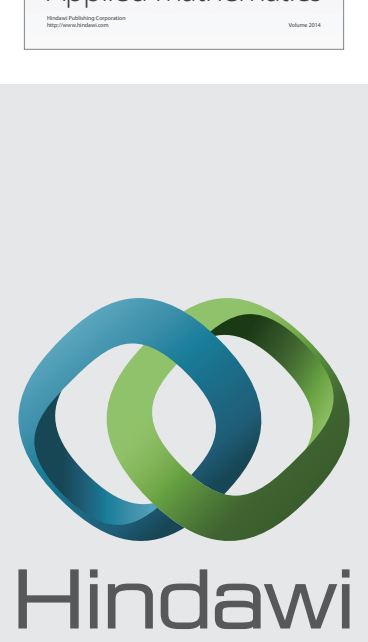

Submit your manuscripts at http://www.hindawi.com
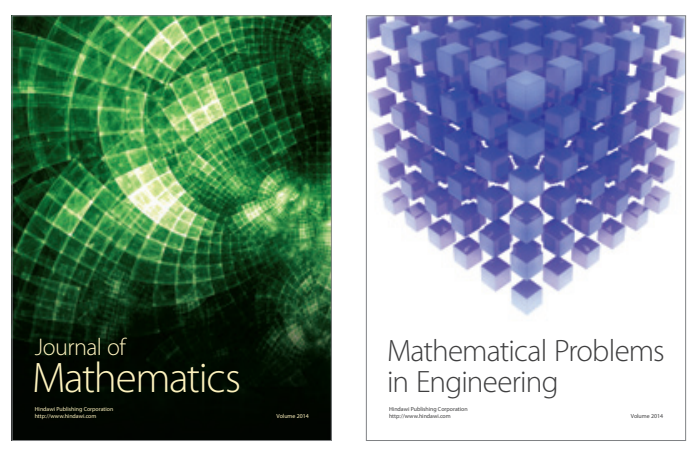

Mathematical Problems in Engineering
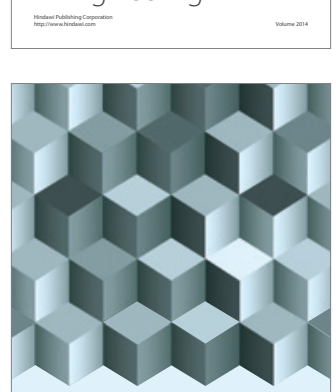

Journal of

Function Spaces
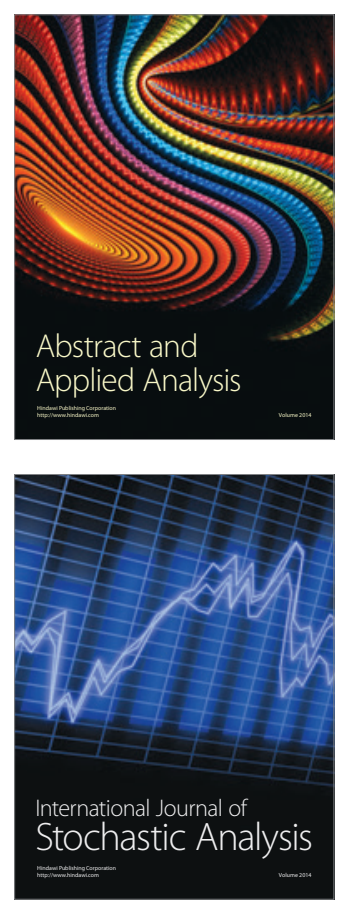

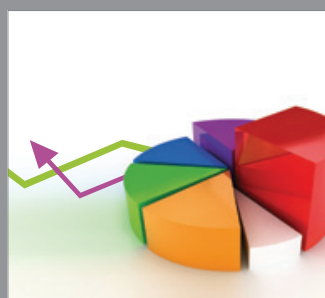

ournal of

Probability and Statistics

Promensencen
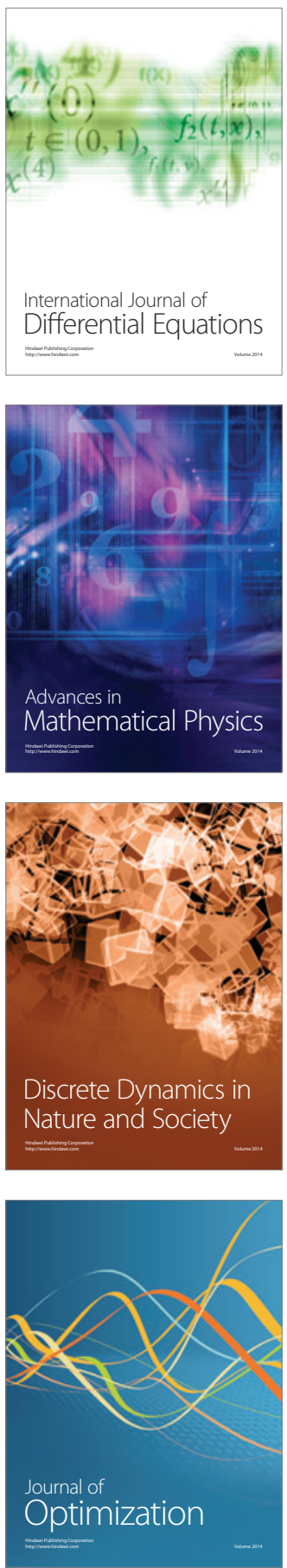